Case Report

\title{
Case report of breast implant-associated anaplastic large cell lymphoma and a call for increased awareness
}

\author{
Brittany Long*, Thushara Dissanayake
}

Department of Surgery, Sunshine Coast University Hospital, Sunshine Coast, Queensland, Australia

Received: 15 January 2022

Accepted: 25 February 2022

\author{
*Correspondence: \\ Dr. Brittany Long, \\ E-mail: Brittany.long@ health.qld.gov.au
}

Copyright: (C) the author(s), publisher and licensee Medip Academy. This is an open-access article distributed under the terms of the Creative Commons Attribution Non-Commercial License, which permits unrestricted non-commercial use, distribution, and reproduction in any medium, provided the original work is properly cited.

\begin{abstract}
Breast implant-associated anaplastic large cell lymphoma (BIA-ALCL) is a rare but serious complication of breast implants. The incidence of BIA-ALCL varies between 1:9121 and 1:13745, and a recent meta-analysis found that countries where specific monitoring measures have been implemented have higher rates of cases. While a significant complication, there is no current evidence to support surgical risk management strategies to prevent BIA-ALCL. Many countries have adopted a model of surveillance, and in Australia the Therapeutic Goods Administration has endorsed data collection through the Australian Breast Device Registry (ABDR). We present a case of a 67-year-old woman who presented to her GP with 2 months of left breast swelling, on a background of a left breast implant in 2004. MRI of the left breast demonstrated a large peri-implant fluid collection, and cytology confirmed BIA-ALCL, CD30-positive and ALK-negative. She underwent a capsulectomy and final histology demonstrated a Breast ImplantAssociated Anaplastic Large Cell Lymphoma with focal penetration of neoplastic cells beyond the capsule and into pericapsular fat (stage T4). While development of registries and national guidelines has ensured the safeguarding of health outcomes for patients moving forward, there is still a concern for women with pre-existing breast implants who are unaware of the risk of BIA-ALCL. This case highlights the importance of raising awareness and providing support to primary health care providers to ensure their patients are given the necessary information to avoid this rare, but serious complication.
\end{abstract}

Keywords: BIA-ALCL, Implants, Breast surgery, Anaplastic lymphoma

\section{INTRODUCTION}

Breast implant associated anaplastic large cell lymphoma (BIA-ALCL) is a rare cancer of the immune system associated with breast implants. ${ }^{1}$ It is characterised by T cells expressing CD30 that are (anaplastic lymphoma kinase) ALK negative. ${ }^{1}$ They arise in close association with breast implants, and typically present as an accumulation of serous fluid between the implant and capsule. $^{2}$

The first case of BIA-ALCL was reported in 1997, and as of May 2021, there are 949 confirmed cases of BIAALCL across 19 countries. ${ }^{3}$ The incidence of BIA-ALCL varies between 1:9121 and 1:13745, and a recent meta- analysis of countries in the European Union found that countries where specific screening measures have been implemented have higher rates of cases, indicating possible under-reporting. ${ }^{4}$

\section{CASE REPORT}

A 67-year-old woman presented to her GP with 6 months of left breast swelling. She had undergone a previous left mastectomy for breast cancer in 1999 with delayed implant reconstruction, and later had an exchange of implant in 2004 to a McGhan 410 FX.

The patient underwent an MRI of the left breast which demonstrated a large volume of $\mathrm{T} 2$ hyperintense fluid 
surrounding the silicone implant. (Figure 1) There was mild enhancement of the external capsule, but no extravasated silicone detected. No suspicious mass or non-mass enhancement was present in the left chest wall, nor was there any axillary lymphadenopathy. Ultrasound guided aspirate was performed and cytology demonstrated Breast Implant-Associated Anaplastic Large Cell Lymphoma, with atypical cells positive for CD30, CD68, GATA3 and CD45, but negative for ALK1, CK7, ER, PR, and MNF116. Preoperative PET CT did not demonstrate any metastatic disease.

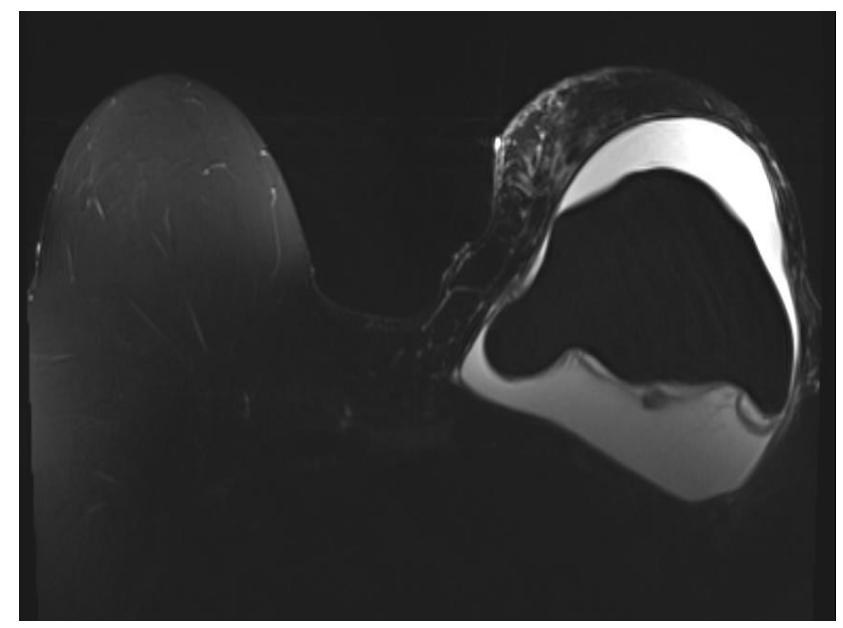

Figure 1: MRI scan in November 2021 of left breast demonstrating a large volume of $\mathrm{T} 2$ hyperintense fluid surrounding a silicone implant.

The patient proceeded to a left capsulectomy and removal of implant and at the time of operation there was no noted capsule rupture or extravasation of silicone. A single axillary lymph node was encountered during the dissection and was sent for histopathology. Final histology of the implant and en bloc capsulectomy demonstrated a Breast Implant-Associated Anaplastic Large Cell Lymphoma with focal penetration of neoplastic cells beyond the capsule and into pericapsular fat (stage T4). The axillary lymph node was negative for neoplasia.

The patient was discussed at the multi-disciplinary meeting and consensus was that further surgery was not indicated, and for follow up with haematology for a bone marrow biopsy and consideration of systemic treatment.

\section{DISCUSSION}

BIA-ALCL is a rare but significant complication of breast implants, and the incidence is increasing exponentially since it was first described. ${ }^{5}$ Proposed theories for the initial low incidence rates include limited registries, lack of awareness, and concerns of litigation. ${ }^{5}$ Worldwide interest and increasing reports of BIA-ALCL, with a developing understanding the pathogenesis of the disease led to multiple countries suspending, cancelling, and recalling implants linked to cases of BIA-ALCL. ${ }^{6}$ In
April 2019, French National Medicines and Health Products Agency (ANSM) suspended multiple textured implants and provided a framework for health-care workers and consumers to raise awareness, and aid reporting. ${ }^{7}$ Countries such as the USA and Australia have since done the same. ${ }^{8,9}$

While the only reported cases of BIA-ALCL have been associated with implants, and a higher risk associated with textured, and larger surface area implants, ${ }^{10}$ there is no current evidence to support surgical risk management strategies, ${ }^{11}$ and the risk of BIA-ALCL is still lower than anaesthetic and surgical risks associated with revision and implant removal. ${ }^{12}$

Increasing incidence of BIA-ALCL also prompted development of registries to ensure adequate documentation and surveillance of implanted devices. In Australia, the Therapeutic Goods Administration (TGA) has endorsed data collection through the Australian Breast Device Registry (ABDR). ${ }^{13}$ The ABDR was established by the Australian Government in 2015 order to track performance, and safety of breast implants, and continued safeguarding of patient outcomes, and currently holds data for more than 36,000 patients. ${ }^{14}$

The TGA website provides up to date evidence of current incidence rates and cases in Australia, and information for consumers and health-care workers. Despite this, there are still consumers who are unaware of the risk of BIA-ALCL, and this lack of awareness and lead to late diagnosis, and risk of progressive disease. In this case, the patient was unaware of BIA-ALCL, or any recall of breast implants, including McGhan 410 FX. $^{15}$ The hospital in which she had her implant exchange had destroyed her records, and the only evidence of her operation report was a copy kept by her surgeon.

BIA-ALCL is becoming an ever-growing concern worldwide, and while the surgical community are well versed in the risk, and remain diligent in continued surveillance, it is not uncommon for patients to lose follow up with their breast surgeons and become reliant on their primary healthcare providers for ongoing care, and surveillance. This case highlights the importance of continued education and support to primary health care providers, and increased exposure of the risk of BIAALCL to current and future consumers.

\section{CONCLUSION}

While development of registries and national guidelines has ensured the safeguarding of health outcomes for patients moving forward, there is still a concern for women with pre-existing breast implants who are unaware of the risk of BIA-ALCL. This case highlights the importance of raising awareness and providing support to primary health care providers to ensure their patients are given the necessary information to avoid this rare, but serious complication. 
Funding: No funding sources

Conflict of interest: None declared

Ethical approval: Not required

\section{REFERENCES}

1. Swerdlow S, Campo E, Pileri S, Harris N, Stein H, Siebert R et al. The 2016 revision of the World Health Organization classification of lymphoid neoplasms. Blood. 2016;127(20):2375-90.

2. Jones J, Hanby A, Wells C, Calaminici M, Johnson $\mathrm{L}$, Turton $\mathrm{P}$ et al. Breast implant-associated anaplastic large cell lymphoma (BIA-ALCL): an overview of presentation and pathogenesis and guidelines for pathological diagnosis and management. Histopathology. 2019;75(6):787-96.

3. Ionescu P, Vibert F, Amé S, Mathelin C. New Data on the Epidemiology of Breast Implant- Associated Anaplastic Large Cell Lymphoma. Eur J Breast Health. 2021;17(4):302-7.

4. Di Pompeo S. Breast Implant-Associated Anaplastic Large Cell Lymphoma (BIA-ALCL): Review of Epidemiology and Prevalence Assessment in Europe. Aesthetic Surg J. 2020;41(9):1014-25.

5. Collett D, Rakhorst H, Lennox P, Magnusson M, Cooter R, Deva A. Current Risk Estimate of Breast Implant-Associated Anaplastic Large Cell Lymphoma in Textured Breast Implants. Plastic and Reconstructive Surgery. 2019;143:30-40.

6. Deva A, Adams W, Vickery K. The Role of Bacterial Biofilms in Device-Associated Infection. Plastic and Reconstructive Surgery. 2013;132(5):1319-28.

7. Vaysse C, Laurent C, Ysebaert L, Chantalat E, Chaput B. France: The First Country to Ban a Type of Breast Implant Linked to Anaplastic Large Cell Lymphoma. Aesthetic Surg J. 2019;39(8):352-53.

8. FDA issues safety communication on Textured Allergan Breast Implants. U.S. Food and Drug Administration. 2022. Available at: https://www.fda.gov/medical-devices/safetycommunications/fda-requests-allergan-voluntarilyrecall-natrelle-biocell-textured-breast-implants-andtissue\#list. Accessed on 15 February 2022.
9. Update-Suspended breast implant devices now cancelled. Therapeutic Goods Administration (TGA). 2022. Available at: https://www.tga.gov.au/alert/breast-implants-andanaplastic-large-cell-lymphoma. Accessed on 15 February 2022.

10. Deva A, Adams W, Vickery K. The Role of Bacterial Biofilms in Device-Associated Infection. Plastic Reconstructive Surg. 2013;132(5):1319-28.

11. Nelson J, McCarthy C, Dabic S, Polanco T, Chilov M, Mehrara B et al. BIA-ALCL and Textured Breast Implants: A Systematic Review of Evidence Supporting Surgical Risk Management Strategies. Plastic Reconstructive Surg. 2021;147(5S):7-13.

12. Breast implant associated cancer (BIA-ALCL): Information for consumers. Therapeutic Goods Administration (TGA). 2022. Available at: https://www.tga.gov.au/breast-implant-associatedcancer-or-bia-alcl. Accessed on 15 February 2022.

13. TGA statement on breast implant and cancer linkAustralian Breast Device Registry. Australian Breast Device Registry. 2022. Available at: https://www.abdr.org.au/tga-statement-breastimplant-cancer-link/. Accessed on 15 February 2022.

14. Breast implant hub. Therapeutic Goods Administration (TGA). 2022. Available at: https://www.tga.gov.au/hubs/breast-implants. Accessed on 15 February 2022.

15. Allergan Issues Worldwide Recall of Biocell Textured Breast Implants and Tissue Expanders. Breastcancer.org. 2022. Available at: https://www.breastcancer.org/researchnews/allergen-recalls-biocell-texturesimplants\#: :text=Recalled $\% 20$ products,FL\%2C\%20 FM\%2C\%20FF\%2C\%20FX. Accessed 15 on February 2022.

Cite this article as: Long B, Dissanayake T. Case report of breast implant-associated anaplastic large cell lymphoma and a call for increased awareness. Int Surg J 2022;9:740-2. 